\title{
How to Reach a Wider Audience with Open Access Publishing: What Research Universities can Learn from Universities of Applied Sciences
}

\section{Saskia Woutersen-Windhouwer}

University of Amsterdam, The Netherlands

s.windhouwer@uva.nl, orcid.org/0000-0003-0120-266X

\section{Jaroen Kuijper}

Amsterdam University of Applied Sciences, The Netherlands j.r.kuijper@hva.nl

\begin{abstract}
In Amsterdam, the libraries of the University of Amsterdam (UvA) and the Amsterdam University of Applied Sciences (AUAS) cooperate closely. In this cooperation, the differences between a research university (i.c. UvA) and a university of applied sciences (i.c. AUAS) become particularly clear when we look at the aim and implementation of open access policies. The open access plan of the AUAS removes not only financial and legal barriers, but also language barriers. This makes the research output FAIR (findable, accessible, interoperable and reusable) to the primary target group of the product, and more importantly, it enables interaction between the AUAS and a wide audience, consisting of researchers from other disciplines, and a wide range of professionals, enterprises, civil servants, schools and citizens. In the search for cofinancing by enterprises and other stakeholders, and to fulfil their valorisation requirements, these target groups are currently becoming more important for research universities as well. Here, we show what research universities can learn from the open access policy of the AUAS.
\end{abstract}

Key Words: university; open access; dissemination; valorization 


\section{Introduction}

According to ROARMAP, the number of research organisations (e.g. universities or research institutions) with an open access policy has steadily grown from 77 in 2005 to 666 in 2017 (ROARMAP, 2006). In particular, after the Berlin Declaration came into being in 2003 (Berlin Declaration, 2003) the number of open access policies started to grow. The policies vary over time. Nowadays, some of the policies are in line with recent policy guidelines, such as the European Commission's policy regarding Horizon2020 (European Commission, 2017) and the recent announcement by European ministers that set the target of $100 \%$ open access by 2020 (Council of the European Union, 2016). Also, the policies of international research funders such as the Bill and Melinda Gates Foundation (Bill \& Melinda Gates Foundation, 2015), and national funders such as the National Institute of Health (NIH, 2016) and the Netherlands Organisation for Scientific Research (NWO, n.d.) influence the open access policies and practices of the universities. All these policies aim to make the information widely and readily available to society: "Our organizations aim to find solutions that support further development of the existing legal and financial frameworks in order to facilitate optimal use and access." (Berlin Declaration, 2003). In general, these open access policies of research organisations focus on the legal and financial aspects, but not on the language aspect, although this is also important for optimal use and access.

It is expected that in the next few years the majority of scientific information will be made widely and readily open access available to society by overcoming the legal and financial barriers (Morrison, 2017). This corresponds with the trends shown by Piwowar et al. (2017) However, another important barrier, the language barrier, is missing in the current open access policies. That is surprising because open access policies also aim to provide open access to a nonacademic audience, whereas most of these publications are written for and can in general only be understood by hyper-specialized academics. As a consequence, non-specialists (including academics, professionals, and the general public) will not be able to easily find or read the information. This is also the main conclusion of the report by the Advisory Council for Science, Technology and Innovation in the Netherlands: according to this report, all scientific publications are already open access available, and we should invest more effort in making the knowledge understandable to non-specialists. "To make scientific results more accessible to society," says the Advisory 
Council, the author must present these results "clearly and in a way that can be understood by a wider public." They also conclude that access to scientific knowledge for business should be better organised (AWTI, 2016). In an article about the future of open access, Richard Price mentions language and jargon barriers too (Price, 2017). He suggests to deliver the same message in different forms. Besides the hyper-specialized article, authors should also provide publications for a general academic audience and the general public. To publish scientific publications truly open access, authors of these publications should also bear the language barrier in mind. When the language barrier has been removed, the knowledge valorisation and distribution to a wider audience will improve considerably.

The meaning of 'valorisation' has changed over time. In the Netherlands, the focus was initially on how the industry could use the knowledge. To stimulate this development the Dutch national funder NWO has grants that can only be submitted if a part is financed by an enterprise. Later any knowledge transfer, also to citizens, was seen as a knowledge valorisation. This fits in with the revision of the Dutch universities of their standard evaluation protocol. The previous protocol mainly evaluated research quality (KNAW, 2009). Nowadays, besides the research quality, also relevance to society will be evaluated (KNAW, 2016). The latest development in the Netherlands is the initiative of the government to democratize the research agenda by means of a National Research Agenda (Dutch national research agenda, 2016), in which all citizens could propose research questions. All these national developments in the Netherlands fit with the Responsible Research and Innovation (RRI) program of the European Commission (EC). RRI was started in 2001 with the Science \& Society Action Plan to improve the connection between the scientific community and society. In the 2007 Science in Society Plan was launched to foster engagement and dialogue between the scientific community and society. And since 2010 RRI means that societal actors work together during the whole research and innovation process; and that the research questions are driven by the needs of society (DirectorateGeneral for Research and Innovation, 2012, 2014). And last but not least, research evaluations and funders more and more demand co-financing by enterprises \& involving professionals, citizens and other stakeholders. For researchers at research universities these initiatives and policies have led to the need to search for co-financing by enterprises, and to involve other stakeholders, for example citizens, the government and other professionals. 
Valorisation and collaboration policies make it really necessary that researchers make knowledge not only open access but also clear and comprehensible to non-specialists.

The Universities of Applied Sciences (UAS) conduct practically oriented research for professionals, education and society, and always address realworld problems. The research is done in close collaboration with academics and professionals. That is why the UAS in the Netherlands have extensive experience with the dissemination of research results to a wider audience. At the Amsterdam University of Applied Sciences (AUAS) about 50\% of the results are written for non-academics (see also Table 1). The AUAS often does advisory work for business and the governmental institutions. At the University of Amsterdam (UvA) this occurs much less often. In the Netherlands UAS differ from Research Universities (RU). RU in the Netherlands have a long tradition in research and education, are more theory-based (focus more on 'why'), and primarily transfer their results to fellow scientists, whereas UAS have mainly had a tradition of teaching, have more practice-oriented education (focus more on 'how'), and offer higher professional education to prepare students for a specific profession such as hotel manager, game designer or nurse (Nuffic, 2018). About 10 years ago, some UAS in the Netherlands have set up applied research activities. In principle, these research activities at the UAS are demand-driven by enterprises and professionals. Due to new valorisation policies, the traditional target groups of the UAS have also become important for researchers at RU. Researchers at UAS already know that (to reach a wider audience) open access is not enough: they are well aware of the language problem when they communicate to non-specialists. Researchers at a RU can therefore learn from researchers at a UAS (who traditionally collaborate with professionals and enterprises) and what they do to make the information better findable and comprehensible to a wider audience.

Table 1: \% of output published in 2016/2017 based on the target group.

\begin{tabular}{lrr}
\hline & AUAS & UvA \\
\hline Academic & $46 \%$ & $80 \%$ \\
Professional \& popular & $54 \%$ & $20 \%$ \\
\hline
\end{tabular}




\section{Open Access Policy of the Amsterdam University of Applied Sciences}

In Amsterdam we have the unique situation that the RU, the University of Amsterdam (UvA), and the UAS, the Amsterdam University of Applied Sciences (AUAS) have different boards, but together have one joint library. Most people who work in the library, work for both institutions. In particular, the open access service owner works for both the UvA and the AUAS, which represent the abovementioned different cultures of a RU and a UAS. The advantage of this situation is that the UvA RU library personnel can learn from their AUAS colleagues, especially when it concerns publication traditions, dissemination and related policies.

In 2015 the Executive Board of the AUAS adopted a Strategic Research Policy for 2015-2020 (Hogeschool van Amsterdam, 2015a) with the following targets for open access: $60 \%$ of scientific publications to be published in open access journals by 2019 and 100\% by 2024; and 100\% of professional publications via the AUAS repository/HBO Knowledge Base ${ }^{1}$ by 2014. In order to meet these targets as soon as possible, the Library UvA/HvA had been asked by the board of the AUAS to formulate an implementation plan for the open access policy. The Library UvA/HvA already had a great deal of knowledge and facilities in the field of open access publishing via the CRIS and the repository of the UvA. Besides the aim to meet the open access targets, with this implementation plan the Library also wanted to increase the visibility of research to potential national and international partners, because this creates more opportunities for collaboration and networking (Vereniging Hogescholen, 2015), and which fits in with the recommendations in the 2015 External Evaluation of AUAS Research Report. This report had recommended a greater focus on Centres for Applied Research and collaboration with other such centres in Europe (Hogeschool van Amsterdam, 2015b). Finally, the Library UvA/HvA also wanted to make sure that in the future it would be easy to connect with the National Knowledge Platform for Applied Research, ${ }^{2}$ whose dual objective is to promote and facilitate knowledge sharing, and to boost the visibility of applied research. The platform emphasises that, for both objectives, it is important that data is shared and connected nationally, rather than just locally or regionally.

With the above policies and recommendations in mind, the Library UvA/ AUAS decided to write a concept open access plan for the AUAS based on 
the FAIR-principle: 'Findable', 'Accessible', 'Interoperable' and 'Reusable' (Wilkinson, et al., 2016; Woutersen-Windhouwer, 2017). The aim of the plan was to make the output of the AUAS easily findable by free search engines and harvesters, accessible by making the output open access by removing financial barriers and also by removing the language barrier, interoperable by using $\mathrm{OAI}-\mathrm{PMH}$ and reusable by removing legal barriers by providing a Creative Commons license. Because the AUAS is copyright owner of the output of its employees, all products can in principle be granted a Creative Commons attribution license, ${ }^{3}$ unless the publisher requests a waiver via one of the AUAS-authors. Terms will then be negotiated by the Library UvA/HvA. To make the implementation plan work, all research results must be registered in the Current Research Information System (CRIS) and archived in the corresponding repository, making them permanently accessible and optimally findable and reusable to third parties. Depositing output in the repository is mandatory (but not necessarily open access) and has been made a part of the research evaluation. Research has shown that only policies that mandate a deposit have a reasonable amount of open access, as the source material must be available to be able to publish open access (Rentier, 2015; Swan, Gargouri, Hunt, \& Harnad, 2015). In this way, the CRIS not only helps to ensure correct, comprehensive and timely registration, but also contributes to the distribution and open sharing of publications. The plan also contains an open access fund and a new AUAS Open series created by open source software that can easily create a pdf, e-pub and html5 which are easy to read on modern devices such as tablets and smart phones. ${ }^{4}$ The implementation plan has been approved by the Centres for Applied Research, the Research Council and the Executive Board of the AUAS in 2016, and will be executed by the Library UvA/HvA, the AUAS Centres for Applied Research, the AUAS Legal Affairs and the AUAS Education and Research Office. The reason that this ambitious plan to go for $100 \%$ open access has been approved, is that in general there is not yet a publication tradition at the AUAS where research activities have started only 10 years ago. In contrast with a RU most results from AUAS are not targeted at an academic audience but at professionals and citizens (see Table 1). As a consequence, at the AUAS, researchers and boards were immediately enthusiastic about open access.

Researchers at the AUAS find it most important that output of their research is optimally accessible, disseminated and reused by academics, professionals, enterprises, scientists, schools and other stakeholders, etc. To facilitate this, 
authors can make use of an open access fund, publish without any costs in the AUAS Open Series, and easily register and upload all their research output (ranging from manuscripts to videogames) in the CRIS combined with a repository. The UvA/AUAS Library has developed an optimally simplified procedure for authors to offer files (publications, reports, games, presentations, etc.) to the AUAS's institutional repository via the CRIS. After registration in the CRIS, the data will be immediately available for all necessary reports (annual reports, research evaluations, etc.). After subsequent validation by one the faculties and the Library the information will be displayed on the staff members' personal pages, and will be easy for everyone to find using resources such as the HBO Knowledge Base, Narcis, Google (Scholar), etc. The data can also be easily exchanged, as the interoperability with other systems is conducted via openly documented APIs and metadata standards (such as Dublin Core, ISNI and ORCID). In addition, in the CRIS authors will also be asked to provide the publication with a Dutch and English layman summary and keywords that are understandable to a wider audience. In the AUAS Open Series, authors are also requested to include a list explaining the terms used, a list of research results (publications, reports, tools), a list of media publications, a reference list, etc. to ensure that all interested parties can understand the core results. This information will help making the information, as mentioned in the report (AWTI, 2016), truly accessible to a wide audience, including non-specialists. The workflow at the AUAS will help to make the research output FAIR to everyone, but more importantly, it will enable interaction between the AUAS and a wide audience, consisting of researchers from other disciplines, and a wide range of professionals, enterprises, civil servants, schools and citizens.

\section{Different Types of Universities, Different Open Access Policies}

The differences between the RU (UvA) and UAS (AUAS) become particularly clear when we look at the aim and implementation of the open access policies of these institutions. The RU in the Netherlands (including the UvA) focus on hybrid open access, which means to transform the traditional tollaccess peer-reviewed articles on the website of the publisher. Big deals with publishers are used to flip from the subscription model to a prepaid open 
access model: the money previously paid for the subscriptions is used for open access publishing to prevent double dipping (Heijne \& van Wezenbeek, 2017; VNSU, 2016). As we have seen, on the contrary, at the AUAS hybrid is not a part of the current policy: the AUAS has only one big deal with a large publisher (and this publisher actually refused to make the AUAS a part of the agreement it had made with the Dutch Universities). To prevent double dipping the AUAS cannot flip existing deals, and hence their open access fund is available only for pure gold open access (as listed in the Directory of Open Access Journals). In contrast to the AUAS, the UvA has no open access fund for authors, does not publish an open access series, and has no policy to stimulate (green) manuscript deposits to be able to publish them in open access.

The reasons behind the difference in open access policies between a RU and a UAS are many. As already mentioned, RU have a long publication tradition, whereas the publication tradition at the UAS is still developing. An important difference is the obsession of the RU with impact factors. Although the RU have a new evaluation protocol and have signed the DORA principles (DORA, 2012) in order to be freed from the impact factor ruling, in practice this situation has not changed yet. The new evaluation protocol should have discouraged quantitative analyses, but we still see a lot of quantitative analyses instead of qualitative. And of course, in RU in a lot of disciplines, careers only go up when the researcher has an article in a high impact journal, and it will also help to get a tenure track position and funding. Other kinds of outputs, such as reports, in principle cannot be counted as scientific. As such these reports do not contribute to the research quality but 'only' to the relevance to society. These aspects are related to a difference in research evalutation: in the Netherlands, researchers at the UAS are evaluated on all output on all levels: peer reviewed academic, professional and popular. That is why the focus on impact factors is smaller at a UAS. It is also important that the target groups of the UAS were always largely outside the scholarly system (enterprises, professionals). Interestingly, as we have seen, these groups are nowadays also more of interest to researchers at RU. Another important difference is that in principle, unless otherwise accepted, the UAS is the copyright owner of the output created by an employee, and not the author. The AUAS does in principle not agree that the copyright will be transferred. If copyright has been transferred, the AUAS keeps the right to publish articles directly in open access in the repository under a CC-BY-license. However, publishers can ask waivers, in principle for a maximum of 6 months. For 
the maximum embargo of 6 months the AUAS refers authors to the Dutch Copyright Act, article 25 fa DCA: within a reasonable term, a researcher may publish a short work open access in a repository. For more extensive publications the AUAS will have to experiment. Finally, it is important for the AUAS open access policy that researchers of the AUAS have stressed that the output from their research should be optimally accessible, disseminated and reused by professionals, enterprises, scientists, schools and other stakeholders.

Researchers at the AUAS know from their own experience how difficult it is to find and access most of the peer reviewed scientific literature. Due to these circumstances, they are more or less in the same situation as most of their stakeholders. The AUAS has access to some important scientific databases and publishers, but most UAS in the Netherlands do not have access to the bibliographic databases such as Web of Science or Scopus, and are dependent on free search engines on the internet. What is more, just like their potential readers and collaborators, they have few or no subscriptions to peer reviewed academic journals. Individual articles can be quite expensive to purchase, and in the end, after paying to see the content, sometimes disappointing. For these reasons, open access is essential for these UAS and their stakeholders. Besides access, information should also be (made) comprehensible for nonspecialists. Authors will therefore be requested to provide together with the results registered in the CRIS a layman summary in Dutch and English and keywords. As most researchers and the boards of the UAS know how difficult it is to get easy access to scientific and comprehensive information, the researchers and boards were enthusiastic about the open access implementation plan.

The approach of a UAS to reach out to a broad audience is nowadays also applicable for RU. As we saw in the introduction, in the search for co-financing by enterprises and other stakeholders and to fulfil their valorisation requirements, this broader audience is becoming more important for RU as well. The same three barriers (financial, legal and language) should be removed if researchers at a RU want to reach out to a wider audience, consisting of researchers from other disciplines, and a wide range of professionals, enterprises, civil servants, schools and citizens. Although all three barriers should be removed, for RU it will not always be as easy to remove the barriers as it is for UAS. The financial barrier will be easy to tackle: in most cases researchers at a RU already publish open access in a repository or with the 
publisher. But removing the legal barrier for users, in order to be able to reuse the materials, will be more difficult. Due to academic freedom RU are in general not the owners of the copyright of the output of their researchers. Therefore, a policy which mandates the attribution of a creative commons license to the works of their researchers will be more difficult at a RU. The legal barrier should be coerced by legal means, by law or by contract. Finally, the language barrier (due to the use of hyper-specialized academic language) can be removed as easily as within the AUAS simply by asking or mandating at least a layman summary (both in English and Dutch) and keywords. RU can easily remove this language barrier by promoting this aspect or even put it into a policy. Adding it to the workflow in a CRIS will help researchers to bear in mind knowledge valorisation and distribution to a wider audience. In the end, removing the language barrier will help RU to reach a wider audience, and it will help researchers to find co-financing, collaborators and to fulfil their valorisation requirements.

\section{Conclusion}

In the past years, most open access policies have focused on the financial and (if possible) on the legal barriers to be able to provide easy access and reuse of information. Although most open access policies mention how important it is to provide this access for everyone, little attention has been paid to making the content of the information also understandable to a wider public and not only for the hyper specialized academic. With the open access implementation of the AUAS, not only financial and legal barriers to access have been removed, but also the language barrier. This makes the research output FAIR and therefore, easy to find for the primary target group of the product; and more importantly, it enables interactions between the AUAS and a wide audience, consisting of researchers from other disciplines, and a wide range of professionals, enterprises, civil servants, schools and citizens. The message for the research universities is clear: if RU follow the example of the AUAS, and removes the language barrier, they will also be able to interact with a wider audience. Interacting with new stakeholders will help the RU to get projects funded, find new collaborators and improve the societal impact of their research. 


\section{Acknowledgement}

We thank Hilde van Wijngaarden for helping us formulate the policy for the AUAS.

\section{References}

AWTI (2016). Dare to share: The road to accessible science. The Hague: Advisory council for science, technology and innovation (Awti). Retrieved from https://www.awti. nl/binaries/awti/documenten/adviezen/2016/01/20/vertaling-dare-to-share/ Dare+to+share.pdf.

Berlin Declaration on Open Access to Knowledge in the Sciences and Humanities (2003, October 22). Retrieved December 29, 2017, from https://openaccess.mpg.de/ Berlin-Declaration.

Bill \& Melinda Gates Foundation (2015). Bill \& Melinda Gates foundation open access policy. Retrieved December 29, 2017, from https://www.gatesfoundation.org/ How-We-Work/General-Information/Open-Access-Policy.

Council of the European Union (2016). Council conclusions on the transition towards an Open Science system. Retrieved from http://data.consilium.europa.eu/doc/ document/ST-9526-2016-INIT/en/pdf.

Directorate-General for Research and Innovation (2012). Responsible research and innovation. Europe's ability to respond to societal challenges. European Commission, EU Publications. https://doi.org/10.2777/11739.

Directorate-General for Research and Innovation (2014). Responsible research and innovation. Europe's ability to respond to societal challenges. European Commission, EU Publications. https://doi.org/10.2777/74572.

DORA (2012). The San Francisco declaration on research assessment. Retrieved December 29, 2017, from https://sfdora.org/read/.

Dutch National Research Agenda (2016). Retrieved December 29, 2017, from http:// www.wetenschapsagenda.nl/national-science-agenda/?lang=en.

European Commission (2017). H2020 Programme - Guidelines to the rules on open access to scientific publications and open access to research data in Horizon 2020, version 3.2. Retrieved from http://ec.europa.eu/research/participants/data/ref/h2020/ grants manual/hi/oa pilot/h2020-hi-oa-pilot-guide en.pdf.

Heijne, M.A.M., \& van Wezenbeek, W.J.S.M. (2017). The Dutch approach to achieving open access. Bibliothek: Forschung und Praxis, 42(1), 36-41. Retrieved from https://doi.org/10.18452/18646. 
Hogeschool van Amsterdam (2015a). Strategisch onderzoeksbeleid 2015-2020. Retrieved from http://www.hva.nl/binaries/content/assets/hva/assets 10/strategischonderzoeksbeleid-2015-2020.pdf.

Hogeschool van Amsterdam (2015b). Rapport externe evaluatie onderzoek 2015 Hogeschool van Amsterdam. Retrieved from https://www.hva.nl/binaries/content/ assets/hva/onderzoek/rapport externe evaluatie hva kenniscentra.pdf.

KNAW (2009). Standard evaluation protocol 2009-2015. Protocol for research assessments in the Netherlands. Koninklijke Nederlandse Akademie van Wetenschappen. Retrieved from https://www.knaw.nl/nl/actueel/publicaties/ standard-evaluation-protocol-sep-2009-2015.

KNAW (2016). Standard Evaluation Protocol 2015-2021. Protocol for research assessments in the Netherlands, Amended version, 2016. Koninklijke Nederlandse Akademie van Wetenschappen. Retrieved from https://www.knaw.nl/nl/actueel/publicaties/ standard-evaluation-protocol-2015-2021.

Morrison, H. (2017, September 30). Dramatic growth of open access series [blog]. Retrieved December 29, 2017, from http://poeticeconomics.blogspot.nl/2006/08/ dramatic-growth-of-open-access-series.html.

NIH (2016). NIH Public access policy details. U.S. Department of Health \& Human Services. Retrieved from https://publicaccess.nih.gov/policy.htm.

NWO (n.d.). Open Science. Netherlands Organisation for Scientific Research.

Retrieved December 29, 2017, from https://www.nwo.nl/en/policies/open+science.

Nuffic (2018). Dutch higher education. Retrieved from https://www.studyinholland. nl/education-system/dutch-higher-education.

Piwowar, H., Priem, J., Larivière, V., Alperin, J.P., Matthias, L., Norlander, B., ..., Haustein, S. (2017). The State of OA: A large-scale analysis of the prevalence and impact of Open Access articles. PeerJ, 6, e4375 (1-23). https://doi.org/10.7717/ peerj. 4375 .

Price, R. (2017, February 13). The evolution of open access [blog]. Retrieved from https://www.huffingtonpost.com/quora/the-evolution-of-open-acc b 14727568. $\underline{\text { html. }}$

Rentier, B. (2015). What next for open access policy? [slideshow] Retrieved from http:// www.pasteur4oa.eu/sites/pasteur4oa/files/generic/Rentier\%20ORBi.pdf.

ROARMAP (2006). The registry of open access repository mandates and policies. Retrieved December 29, 2017, from http://roarmap.eprints.org.

Swan, A., Gargouri, Y., Hunt, M., \& Harnad, S. (2015). Report on policy recording exercise, including policy typology and effectiveness and list of further policymaker targets.

D 3.1 PASTEUR4OA. Retrieved from http://www.pasteur4oa.eu/sites/pasteur4oa/ 
files/deliverables/PASTEUR4OA $\% 20$ Work $\% 20$ Package $\% 203 \% 20$ Report $\% 20$ final $\% 20$ 10\%20March $\% 202015$.pdf.

Vereniging Hogescholen (2015). Brancheprotocol Kwaliteitszorg Onderzoek 20162022. Kwaliteitszorgstelsel Praktijkgericht Onderzoek Hogescholen. Retrieved from http://www.vereniginghogescholen.nl/system/knowledge base/attachments/ files/000/000/489/original/BKO 2016-2021 - okt 2015 (12-1-2016 definitief).pdf.

VNSU (2016). The Netherlands: paving the way for open access. Vereniging an universiteiten. Retrieved December 29, 2017, from http://www.magazine-on-thespot.nl/openaccess/eng/.

Wilkinson, M.D., Dumontier, M., Aalbersberg, I.J., Appleton, G., Axton, M., Baak, A., ..., Mons, B. (2016). The FAIR guiding principles for scientific data management and stewardship. Scientific Data 3(160018), 1-9. https://doi.org/10.1038/sdata.2016.18.

Woutersen-Windhouwer, S. (2017). AUAS research results: Open, findable and reusable. Step-by-step plan v2.1 (public version). Hogeschool van Amsterdam. Retrieved from http://www.amsterdamuas.com/binaries/content/assets/subsites/hvabibliotheek/hva-stappenplan-open-publiceren-publieke-versie v2.1-en-bewerkt.pdf.

\section{Notes}

${ }^{1}$ The HBO Knowledge Base offers public access to research results from Dutch Universities of Applied Sciences.

${ }^{2}$ A plan has been created for the future merger of the HBO Knowledge Base with the Podium of the SIA National Regional Body for Practical Research (Nationaal Regieorgaan Praktijkgericht Onderzoek SIA), which will continue under a new name. The infrastructure will be maintained, but more attention will be paid to promoting and marketing it to researchers in order to increase the number of publications deposited in the knowledge base. Plans are also being devised for active editing within the new platform, and maybe even an editing network enabling the use of regional differences and networks of disciplines.

${ }^{3}$ https://creativecommons.org/licenses/by/4.0/legalcode.

${ }^{4}$ http://www.publishinglab.nl/hvaopen/2016/09/01/

hva-open-publishing-introduction/. 